\title{
Foldamer-Based Ultrapermeable and Highly Selective Artificial Aquaporins that Exclude Protons
}

\author{
Arundhati Roy, ${ }^{1, \ddagger}$ Jie Shen, ${ }^{1, \ddagger}$ Himanshu Joshi, ${ }^{2}$ Woochul Song, ${ }^{3}$ Yu-Ming Tu, ${ }^{3}$ Ruijuan Ye, ${ }^{4}$ Ning Li, ${ }^{1}$ Changliang Ren, ${ }^{1}$ \\ Manish Kumar, ${ }^{5}$ Aleksei Aksimentiev, ${ }^{2}$ and Huaqiang Zeng ${ }^{1, *}$
}

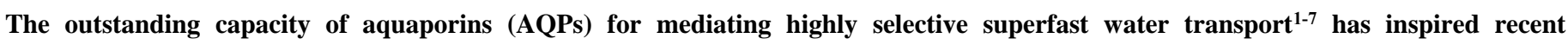
development of supramolecular monovalent ion-excluding artificial water channels (AWCs). AWC-based bioinspired membranes are proposed for desalination, water purification, and other separations applications ${ }^{8-18}$. While some recent progress has been made in synthesizing AWCs that approach the water permeability and ion selectivity of AQPs, a hallmark feature of AQPs - high water transport while excluding protons has not been reproduced. We report on a class of biomimetic, helically folded pore-forming polymeric foldamers, that can serve as long sought-after highly selective ultrafast water-conducting channels exceeding those of $\mathrm{AQPs}^{(1.1} \times 10^{10} \mathrm{H}_{2} \mathrm{O}$ molecules/s for $\left.\mathrm{AQP1}{ }^{7}\right)$, with high water over monovalent ion transport selectivity $\left(\sim \mathbf{1 0}^{8}\right.$ water molecules over $\mathrm{Cl}^{-}$ion) conferred by the modularly tunable hydrophobicity of the interior pore surface. The best-performing AWC reported here delivers water transport at an exceptionally high rate, 2.5 times that of $\mathrm{AQP1}$, while concurrently rejecting salts ( $\mathrm{NaCl}$ and $\mathrm{KCl})$ and even protons.

Drawing on Nature for inspiration, biomimicry has provided rich and effective technological solutions to a range of complex problems ${ }^{19}$. One challenging line of inquiry in biomimicry is the extent to which man-made materials can mimic Nature or even go beyond the boundaries seen in Nature at the macro and nanoscales. At the molecular level, biological membrane transport channels play indispensable roles in myriad cellular processes via precisely controlled directional flows of cognate species (including water ${ }^{1-7}$, potassium $^{20}$, sodium ${ }^{21}$, calcium ${ }^{22}$, chloride ${ }^{23}$, and protons ${ }^{24}$. These precisely controlled flows occur through narrow, often water-filled, membrane-spanning pores. Nevertheless, the two highly integrated quintessential features collectively seen in many natural membrane channels - high selectivity that is seamlessly integrated with high permeation rate - are exceedingly difficult to replicate in artificially developed membrane materials ${ }^{12}$ and more specifically artificial channels and carriers. In fact, despite numerous advances made over the past four decades ${ }^{15-18,25-44}$, no artificial channels or membranes have reached the performance in transmembrane transport of ions, protons or water molecules by biological channels such as the KcsA potassium channel and AQPs. The unique ability of AQPs to exclude protons while transporting water decouples water transport from maintenance of transmembrane proton gradients ${ }^{45}$ which is critical to sustaining life in most living cells. This feature has not been previously reproduced in artificial channels or engineered biological channels. Through a minimalist biomimetic approach, we have for the first time succeeded in outperforming $\mathrm{AQP} 1^{1-7}$ in water transport rate while concurrently retaining high rejection of both salts $(\mathrm{NaCl}$ and $\mathrm{KCl})$ and protons.

The archetypal AQP, aquaporin 1 (AQP1), can transport $\sim 1.1 \times 10^{10}$ $\mathrm{H}_{2} \mathrm{O} / \mathrm{s}^{7}$ while excluding all ions. Such outstanding water over ion selective water transport performance, estimated at $>10^{9}$ water molecules over monovalent ions ${ }^{46}$, has inspired the development of next-generation AQP-based biomimetic membranes for industrial and municipal desalting and water purification applications ${ }^{8-18}$. Proteinbased membranes, however, are expected to have a high production cost and face perceived challenges with scale up and stability for industrial uses ${ }^{11}$. These challenges may limit their widespread applications in novel membrane technologies for water purification and other applications including blue energy production, organic solvent nanofiltration and organic solvent reverse osmosis ${ }^{18}$. Aiming to mimic the functions of AQPs and to enable a wider range of molecular separations applications, bioinspired supramolecular artificial water channels (AWCs) have emerged as promising materials. ${ }^{25-34}$ Yet, concurrently attaining high single-channel water permeability and high transport selectivity has proven to be challenging. Indeed, among all hitherto reported AWCs with high selectivity ${ }^{25-33}$, only very recently were self-assembled AWCs reported to reject salts, and transport water at a rate of $3.0 \times 10^{9} \mathrm{H}_{2} \mathrm{O}$ molecules $/ \mathrm{s}^{31,33}$, which is $\sim 27 \%$ that of AQP1. Carbon nanotube porins (CNTPs) with an internal pore diameter of 4.7 $\AA$ exhibit a water conduction rate 2.1 times that of $\mathrm{AQP} 1^{34}$. However, CNTPs also conduct $\mathrm{Na}^{+}, \mathrm{K}^{+}$and protons ${ }^{34,41}$.

\footnotetext{
Molecular design principles and considerations for AWCs We speculated that a large hollow tubular cavity, possessing a pore diameter much larger than a water molecule $(2.5 \AA)$ but still smaller than a sodium ion with its first hydration shell $(\sim 9 \AA$, Supplementary Fig. 1a), might be needed in order to compete with AQPs designed by nature. More precisely, we wanted to further investigate if water transport that does not strictly adhere to the single-file water arrangement seen in AQP1 and other channels ${ }^{32,33,34}$ might be able to enable water transport rates higher than AQP1.

We propose that to attain high salt rejection by these large pores, the functional groups decorating the interior pore surface should be designed to be "incompetent" in replacing water molecules coordinating hydrated ions and thus increasing the barrier to ion entry
}

\footnotetext{
${ }^{1}$ NanoBio Lab, 31 Biopolis Way, The Nanos, Singapore. ${ }^{2}$ Department of Physics and the Center for the Physics of Living Cells, University of Illinois at UrbanaChampaign, Urbana, IL, USA. ${ }^{3}$ Department of Chemical Engineering, The University of Texas at Austin, Austin, TX, USA. ${ }^{4}$ Department of Chemical and Biomolecular Engineering, National University of Singapore, Singapore. ${ }^{5}$ Department of Civil, Architectural and Environmental Engineering, The University of Texas at Austin, TX, USA.

‡ These authors contributed equally to this work. *Corresponding author. Email: hqzeng@nbl.a-star.edu.sg (H.Z.)
} 
a

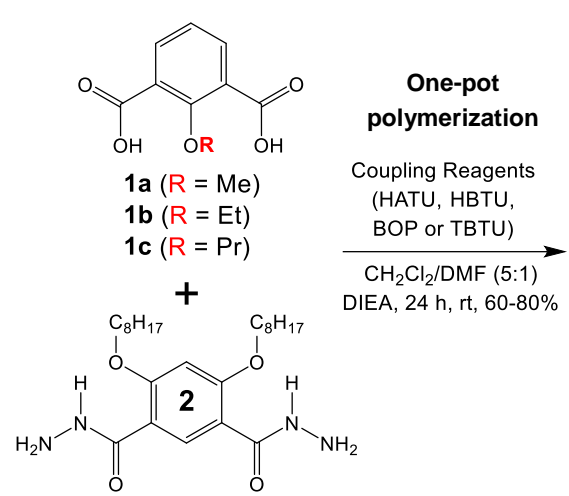

b

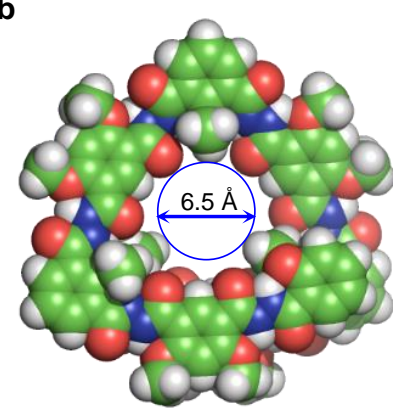

QM-optimized pore scaffolds of channel 3

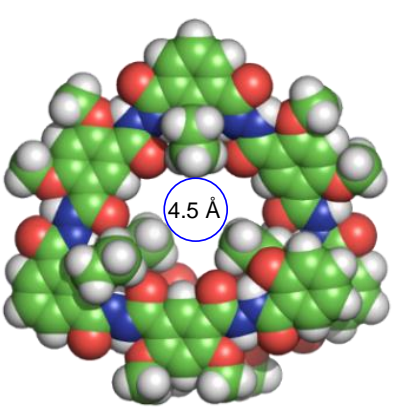

QM-optimized pore scaffolds of channel 4
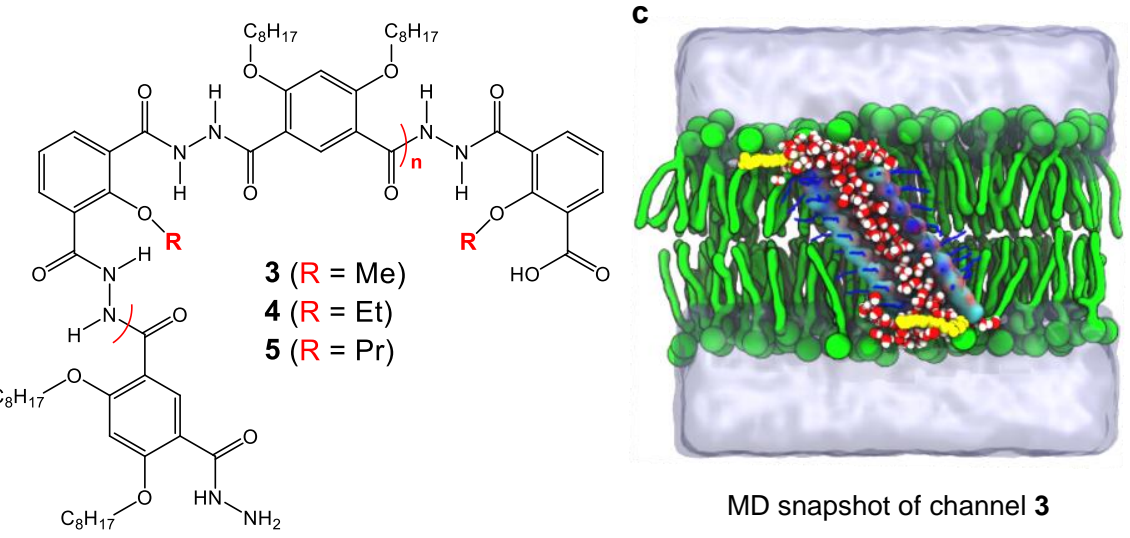

MD snapshot of channel 3

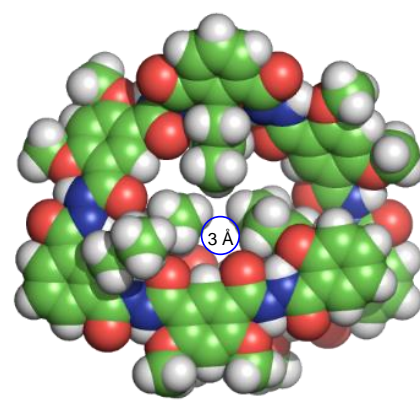

QM-optimized pore Scaffolds of channel 5

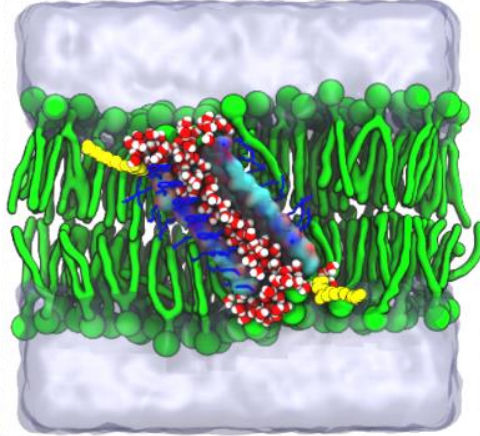

MD snapshot of channel 4

Fig. 1 Molecular design and synthetic construction of foldamer-derived polymer-based synthetic water channels. a, Chemical structures of artificial water channels 3-5, which have inwardly facing methyl- (3), ethyl- (4), or isopropyl- (5) aliphatic groups for tuning the inner-pore size and hydrophobicity, respectively. b, QM-optimized backbone scaffolds of $\mathbf{3}-\mathbf{5}$, which show good structural correspondence to the crystallographic structures of methyl- and ethyl- containing trimeric repeating units (See $\mathbf{6 a}$ and $\mathbf{6 b}$ in Supplementary Fig. $1 \mathrm{~b}$ for details). c, Cross-sections of MD snapshots of water-filled lipid anchor-containing channels $\mathbf{3}$ and $\mathbf{4}$, each containing 26 repeating units 1 and 26 repeating units $\mathbf{2}$ and having a height of $2.9 \mathrm{~nm}$, in POPC membrane. The system is solvated in $1 \mathrm{M} \mathrm{NaCl}$, with the volume occupied by the electrolyte solution represented as semi-transparent surfaces. POPC $=(1$-palmitoyl-2-oleoyl-sn-glycero-3-phosphatidyl choline $)$.

into channels. Strategic distribution of such functional groups in a way that the necessary convergent binding surface for stripping water molecules off the ions becomes less accessible or even inaccessible to the ions would then inhibit ion passage. The overall strategy implemented here further involved use of aliphatic chains to render the cavity hydrophobic. Differing from $\pi$-electron rich hydrophobicity in CNTs that could interact with cations ${ }^{47}$, such "aliphatic hydrophobicity" conferred by the essentially non-polar $\mathrm{C}-\mathrm{H}$ bonds provides a slightly positive electrostatic surface ${ }^{40}$ that excludes cations. With a sufficiently small cavity, we propose that it is highly likely that anion transport through these channels will also be blocked. Moreover, we focused on designs where one-dimensional $\mathrm{H}$-bonded water chains cannot be formed continuously inside the pores. This was achieved by providing conditions for water interaction with the channel where water molecules were not able to transfer protons to adjacent waters via the Grotthuss mechanism and thus blocking proton transport. This mechanism of proton wire disruption is similar to that seen in the NPA motif present in the central section of all $\mathrm{AQPs}^{48}$ and does not seem to significantly disrupt the high water transport rates of AQPs. This work thus also addresses the question of whether continuously formed $\mathrm{H}$ bonded water chains (seen in CNTPs ${ }^{34}$ and other channels ${ }^{32,33}$ ) are absolutely necessary for rapid water transport.

\section{Molecular design and structural features of AWCs 3-5}

We recently reported polymeric anion channels 3 (Fig. 1a) derived from an H-bond-rigidified foldamer-based hydrazide backbone ${ }^{40}$. Arising from hydrophobic methyl groups and well-separated interior O-atoms that can't form a convergent cation-binding pocket (Fig. 1b,d), the large internal hollow cavity of $\sim 6.5 \AA$ in 3 efficiently blocks cation transport while still allowing anion transport. With the above molecular design principles and considerations in mind, interior methyl groups $(\mathrm{Me})$ in 3 were replaced by ethyl (Et) or propyl (Pr) groups to furnish two new classes of polymer channels labelled 4 and $\mathbf{5}$. The elongated and more hydrophobic Et and Pr groups protrude toward the cavity center, thus making the cavity smaller and minimizing the anion-cavity interactions for increased anion rejection.

Quantum mechanics (QM) calculations of pore scaffolds at the level of $\omega \mathrm{B} 97 \mathrm{X} / 6-31 \mathrm{G}(\mathrm{d})$ were first adopted to understand the structural features of nanotubes $3-\mathbf{5}$. The fact that the central fragments of QMcomputed pore scaffolds of $\mathbf{3}$ and $\mathbf{4}$ (Fig. 1b) are nearly-identical to crystal structures of two trimer molecules (see $\mathbf{6 a}$ and $\mathbf{6 b}$ in Supplementary Fig $1 \mathrm{~b}$ for details), respectively, indicates high reliability of computed pore sizes of $\geq 6.5 \AA$ for $3, \geq 4.5 \AA$ for 4 and three segregated pores of $\sim 3 \AA$ for $\mathbf{5}$. Molecular dynamics (MD) simulations were carried out to provide further insights into $\mathbf{3}$ - $\mathbf{5}$ of 2.9 $\mathrm{nm}$ long, each having 26 units of $\mathbf{1}$ and 26 units of $\mathbf{2}$. As expected, both 3 and $\mathbf{4}$ enclose a water-filled sizable cavity of $>4 \AA$ (Fig. 1c), with the cavity of $\mathbf{5}$ mostly blocked by the Pr groups (Supplementary Fig. 1c). 


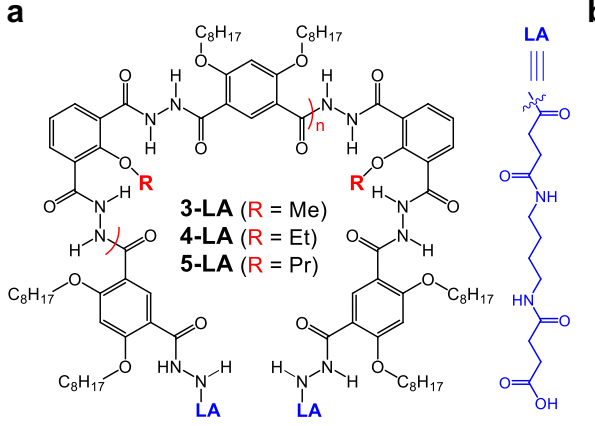

d
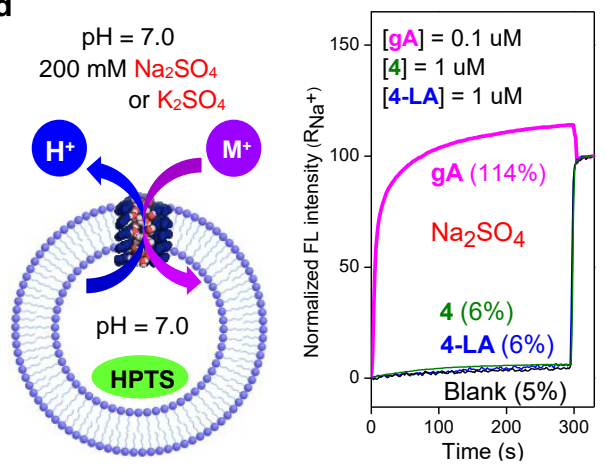

f

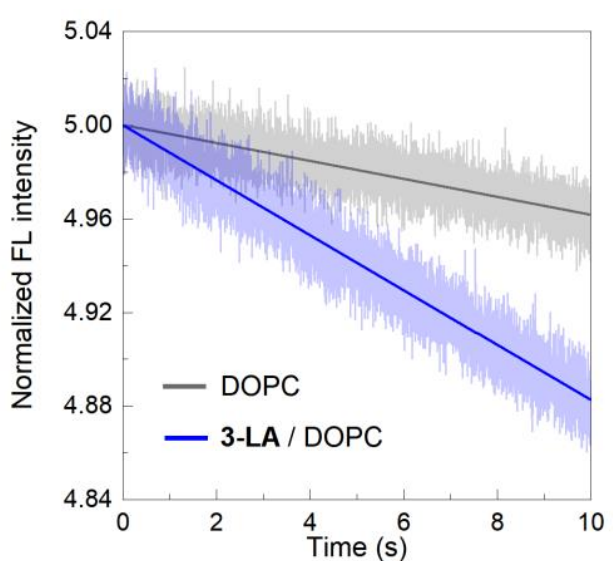

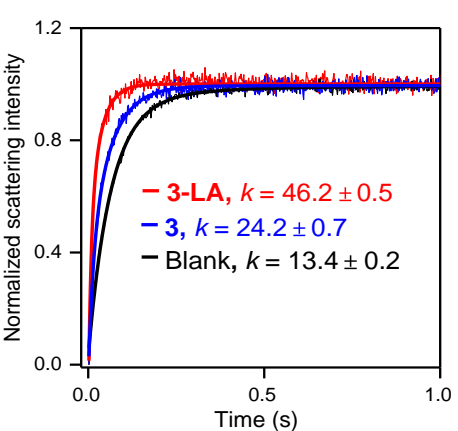

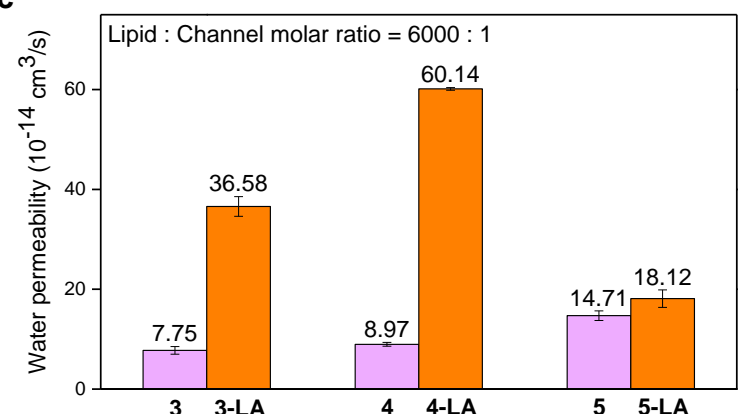

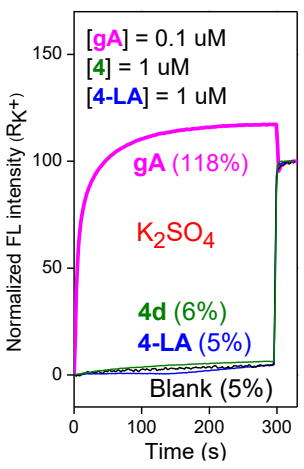

g

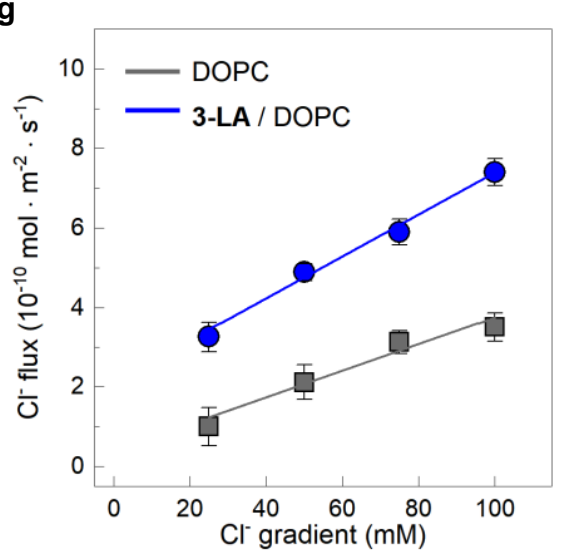

e $\quad \mathrm{pH}=8.0$

$67 \mathrm{mM} \mathrm{Na}_{2} \mathrm{SO}_{4}$

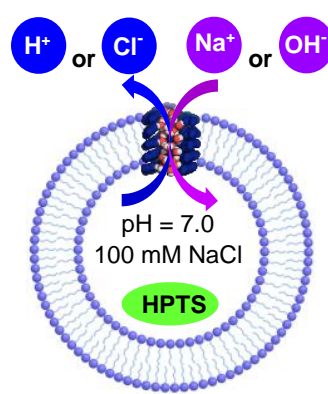

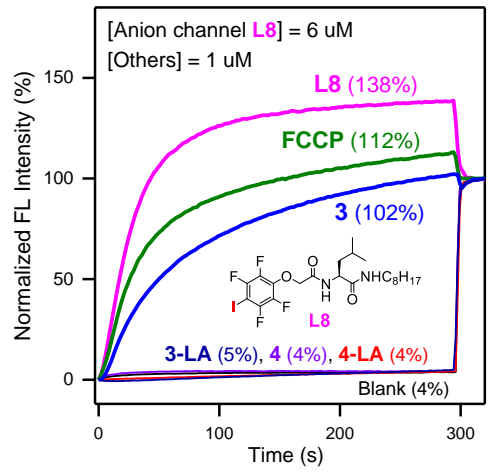

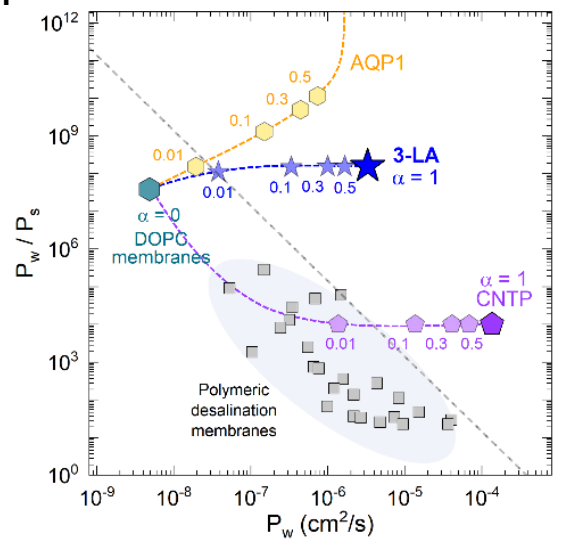

Fig. 2 | Selective water permeation through foldamer-based polymeric artificial water channels. a, Chemical structures of lipid anchor (LA)-modified artificial water channels. b, Representative stopped-flow light scattering traces of $\mathbf{3}$ and 3-LA reconstituted DOPC vesicles under the inwardly directed osmotic gradients. $\mathbf{c}$ Single channel permeability of channels before and after LA modification. $\mathbf{d}$ and $\mathbf{e}$, Schematic illustrations of HPTS-based fluorescence assays, demonstrating high rejection of (d) cations $\left(\mathrm{Na}^{+}\right.$or $\left.\mathrm{K}^{+}\right)$and $(\mathbf{e})$ anion $\left(\mathrm{Cl}^{-}\right)$and proton through the channels. f, Stopped-flow fluorescence traces of 3-LA reconstituted DOPC vesicles, encapsulating lucigenin dyes for sensing $\mathrm{Cl}^{-}$permeation that is driven by inwardly directed $\mathrm{Cl}^{-}$concentration gradients $(20 \mathrm{mM})$. $\mathbf{~ g , ~ P l o t s ~ o f ~ C l - ~ p e r m e a t i o n ~ f l u x ~ a s ~ a ~}$ function of $\mathrm{Cl}^{-}$concentration gradients for both DOPC and 3-LA reconstituted DOPC membranes. h, Comparison of water-to-salt permselectivity of 3-LA to the values of several representative water channels and current polymeric desalination membranes. Intrinsic water-to-salt selectivity $(P w / P s)$ versus water permeability $\left(P_{w}, \mathrm{~cm}^{2} / \mathrm{s}\right)$ plot. $P w / P s$ vs. $P w$ values for 3-LA reconstituted biomimetic membranes in a DOPC matrix, which were compared with other representative water channels, AQP1 and carbon nanotube porins (CNTPs) with pore diameter of $\sim 0.47 \mathrm{~nm}^{13,34}$. The $\alpha$ values in the plot indicate the fractional volume of the biomimetic membranes occupied by water channels (e.g., $\alpha=0.5$ represents the membranes of which the half volume is composed of the channels). Dashed line is the current polymer desalination membranes' permeability-selectivity upper bound limit ${ }^{10,12}$.

\section{High water permeability of AWCs 3-5}

Following a recently reported one-pot polymerization protocol ${ }^{40}$, twelve AWCs $(\mathbf{3}-\mathbf{5})$ were readily made from repeating units $\mathbf{1}$ and $\mathbf{2}$ using coupling reagents (HATU, HBTU, BOP and TBTU, see Fig. 1a and Supplementary Fig. 2 and Supplementary Tables 1-3 for details), with GPC-derived molecular weights $\left(\mathrm{M}_{\mathrm{n}}\right)$ of $11.6-27.3 \mathrm{KDa}$ and nanotube lengths of $2.1-5.1 \mathrm{~nm}$.
Single channel water permeability $\left(P_{s c}\right.$ in $\left.\mathrm{cm}^{3} / \mathrm{s}\right)$ was investigated by a stopped- flow method ${ }^{25-34}$, using $0.3 \mathrm{M}$ sucrose as the draw solution. To facilitate quick identification of effective AWCs, $100 \%$ channel membrane insertion efficiency was initially assumed for all channels. This simple assumption turns out to be highly valid since the actual channel insertion efficiencies are near-identical among $\mathbf{3}-\mathbf{5}$ under the identical conditions (see latter discussion). By varying the lipid to 
channel molar ratios (mLCR) from 1500:1 to 9000:1, 3 of $3.0 \mathrm{~nm}$ long, channels made using HATU as the coupling agent, was found to elicit the maximum permeability at mLCR of 6000:1 (Supplementary Fig. 3 and Supplementary Table 4). Fixing the mLCR to 6000:1, single channel permeability $\left(P_{s c}\right)$ values appear to plateau at a channel height of $3.0 \mathrm{~nm}\left(7.75 \pm 0.76 \times 10^{-14} \mathrm{~cm}^{3} / \mathrm{s}\right)$ among series 3 , of $3.1 \mathrm{~nm}(8.97$ $\left.\pm 0.76 \times 10^{-14} \mathrm{~cm}^{3} / \mathrm{s}\right)$ among series 4 , and of $2.1 \mathrm{~nm}\left(14.72 \pm 0.97 \times 10^{-}\right.$ $14 \mathrm{~cm}^{3} / \mathrm{s}$ ) among series 5 (Supplementary Table 5). These values translate into water transport rates of $\sim 10^{9} \mathrm{H}_{2} \mathrm{O}$ molecules/s.

\section{Ultrapermeability of lipid anchor-modified 4-LA}

We surmised that $\mathbf{3}$ and $\mathbf{4}$ likely could transport water molecules much faster than $\sim 10^{9} \mathrm{H}_{2} \mathrm{O}$ molecules / $\mathrm{s}$ because of their pore sizes which are much larger than a water molecule. Channels 3 and 4 lack hydrophilic end groups for aligning channels to span lipid bilayers seen in other water channels, leading us to hypothesize that many unproductive channel orientations not conducive for water transport may exist. To examine this surmise, carboxylic acid-based "lipid anchors" (LAs, Fig. 2a) were attached at both termini of the most active channels among series 3, 4 and 5 to generate 3-LA, 4-LA and 5-LA to improve channel orientation and water transport activity was evaluated.

Compared to 3 free of lipid anchors, water conduction of 3-LA was improved (Fig. 2b), and its $P_{s c}$ value averaged over three runs was determined to be $36.58 \pm 1.97 \times 10^{-14} \mathrm{~cm}^{3} / \mathrm{s}$ at a mLCR of $6000: 1$ (Fig. 2c). Similarly, $P_{s c}$ values of 4-LA and 5-LA were increased to $60.14 \pm$ $0.17 \times 10^{-14} \mathrm{~cm}^{3} / \mathrm{s}$ and $18.12 \pm 1.76 \times 10^{-14} \mathrm{~cm}^{3} / \mathrm{s}$, respectively. After corrections using the actual channel insertion efficiencies of $66 \%, 75 \%$, and 75\% for 3-LA, 4-LA and 5-LA (Supplementary Fig. 4 and Supplementary Table 6), their respective water conduction rates were determined to be $1.6 \pm 0.06 \times 10^{10}, 2.7 \pm 0.005 \times 10^{10}$ and $0.8 \pm 0.05$ $\times 10^{10} \mathrm{H}_{2} \mathrm{O} / \mathrm{s}$.

The 4-LA channel mediated ultrafast water conduction of $2.7 \times 10^{10}$ $\mathrm{H}_{2} \mathrm{O} / \mathrm{s}$, which represents a large improvement of $145 \%$ over that of AQP1 $\left(1.1 \times 10^{10} \mathrm{H}_{2} \mathrm{O} / \mathrm{s}\right)^{7}$, and is even higher than that of CNTPs $(2.3$ $\left.\times 10^{10} \mathrm{H}_{2} \mathrm{O} / \mathrm{s}\right)^{34}$ at $\mathrm{pH} 7$.

The water transport activation energy values $\left(E_{a}\right)$ for DOPC membrane only, 3-LA, 4-LA and 5-LA were determined to be $12.3 \pm$ $1.3,8.7 \pm 1.2,8.3 \pm 0.9$ and $11.5 \pm 1.2 \mathrm{Kcal} / \mathrm{mol}$, respectively (Supplementary Fig. 5). Consistent with recent observations by us ${ }^{32}$ and others ${ }^{4,33}, E_{a}$ value for more permeable 4-LA, while lower than though pure lipids, turns out to be larger than that of less permeable AQP1 $(\sim 5 \mathrm{Kcal} / \mathrm{mol})^{5}$. This suggests that the low activation energy seen in AQP channels ${ }^{7,33,49}$ may not be a requirement for all highly permeable channels, particularly when the transport is not strictly single water-wire mediated.

\section{High rejection of salts and protons by 4-LA}

Another indispensable criterion of being an ideal AWC concerns the ability to reject salts $(\mathrm{KCl}$ and $\mathrm{NaCl})$ and ideally protons. The ability of 4-LA to reject salts was first evaluated by comparing osmotic water permeability $\left(P_{f}\right.$ in $\mathrm{cm} / \mathrm{s}$ ) values under three hypertonic buffer solutions (300 $\mathrm{mM}$ sucrose, $150 \mathrm{mM} \mathrm{NaCl}$ or $150 \mathrm{mM} \mathrm{KCl}$, Supplementary Fig. 6). At an mLCR of $6000: 1$, the measured $P_{f}$ values for $\mathbf{4 - L A}$ were $117.5 \pm 4.5,121.5 \pm 3.8$ and $124.7 \pm 0.3 \mathrm{~cm} / \mathrm{s}$ for sucrose, $\mathrm{NaCl}$ and $\mathrm{KCl}$, respectively. The corresponding reflection coefficients, defined as the ratio of $P_{f}$ values, are $1.03 \pm 0.01$ for $P_{f}(\mathrm{NaCl}) / P_{f}$ (sucrose) and $1.06 \pm 0.006$ for $P_{f}(\mathrm{KCl}) / P_{f}$ (sucrose), confirming that 4-LA achieves nearly $100 \%$ rejection of $\mathrm{NaCl}$ and $\mathrm{KCl}^{28,32,33}$. These approximate values indicating salt rejection can be corroborated by the low reflection coefficients of $0.53 \pm 0.02$ and $0.07 \pm 0.001$ for cation channel gramicidin $\mathrm{A}(\mathrm{gA})$, which is highly permeable to both $\mathrm{Na}^{+}$and $\mathrm{K}^{+}$ions, for $\mathrm{NaCl}$ and $\mathrm{KCl}$, respectively.

High rejection of $\mathrm{Na}^{+}$and $\mathrm{K}^{+}$was validated further using LUV (large unilamellar vesicle) based assays, with entrapped $\mathrm{pH}$-sensitive HPTS dye (Fig. 2d). Exchange via $\mathrm{H}^{+} / \mathrm{M}^{+}$antiport mechanism was monitored by change of fluorescence intensity of the HPTS dyes in presence of high salt gradient (200 mM Na $\mathrm{SO}_{4}$ or $\mathrm{K}_{2} \mathrm{SO}_{4}$ ). Unsurprisingly, channels 4 and 4-LA at $1 \mu \mathrm{M}$ both are completely inactive towards both $\mathrm{K}^{+}$and $\mathrm{Na}^{+}$ions, whereas dimeric $\mathrm{gA}$, at channel concentrations 20 times smaller $(0.1 \mu \mathrm{M})$, transports both $\mathrm{Na}^{+}(114 \%)$ and $\mathrm{K}^{+}(118 \%)$ at high rates. The presence of 4-LA in the bilayer membrane can be established by the measured membrane insertion efficiency of $\geq 75 \%$ under the identical conditions (Supplementary Fig. 7). Additional testing similarly reveals high cation rejections by $\mathbf{3}, \mathbf{3 - L A}, \mathbf{5}$ and 5-LA (Supplementary Fig. 8a), demonstrating that a pore as large as $6.5 \AA$ in 3 can effectively prevent both $\mathrm{Na}^{+}$and $\mathrm{K}^{+}$from entering it, along with the smaller mostly hydrophobic pores, $\mathbf{4}$ and $\mathbf{5}$.

High rejection of $\mathrm{Cl}^{-}$and protons was confirmed by using the LUV scheme shown in Fig. 2e. Specifically, when compared to a) a selfassembled chloride channel (F8) $6^{50}$ that induces a high chloride transport activity of $138 \%$, and b) the proton carrier FCCP that induces a high proton transport activity of $112 \%$, both the anion and proton transport rates of 3-LA, 4, 4-LA (Fig. 2e), 5, and 5-LA (Supplementary Fig. 8b) were non-detectable in this assay. Our conservative estimate indicates the 4-LA-induced proton transport rate to be $<1 \times 10^{-2}$ proton/s (Supplementary Fig. 9 and the corresponding supplementary discussions), corresponding to high $\mathrm{H}_{2} \mathrm{O} / \mathrm{H}^{+}$selectivity of $>2.7 \times 10^{12}$ at $\mathrm{pH}$ 7. Importantly, a large difference in $\mathrm{Cl}^{-}$transport between 3 and 3-LA suggests a new strategy of using lipid anchors or other end modifiers to eliminate the otherwise high anion transport activity of $\mathbf{3}^{40}$

High rejection of $\mathrm{Cl}^{-}$also can be established by using a chloridesensitive SPQ dye (Supplementary Fig. 8c). Except for 3, channels 3LA, 4, 4-LA, 5 and 5-LA all caused quenching of SPQ dye undifferentiated from the background, whereas (F8) 6 displayed $45 \%$ fluorescence quenching.

Therefore, results from these LUV-based assays prove evidence of high rejection of both salts and protons by ultrapermeable 4-LA and by superpermeable 3-LA, 4, 5 and 5-LA.

To evaluate the molecular separation property of the channels for desalination applications, monovalent ion permeability of 3-LA was measured, and its water-to-salt permselectivity compared to the values of the other representative membrane materials ${ }^{10,12,13,34} \cdot \mathrm{Cl}^{-}$ion permeability was measured using stopped-flow fluorescence analysis, with lucigenin dyes as molecular sensing probes (Fig. 2f), as demonstrated in a recent study ${ }^{31}$. The transport rate obtained from these analyses was used to represent net monovalent ion (e.g., $\mathrm{Na}^{+}$and $\mathrm{Cl}^{-}$) permeability of the channels. This estimate is a conservative method of measuring monovalent ion permeability because, in actual desalination processes, $\mathrm{Cl}^{-}$permeation is limited and lowered by $\mathrm{Na}^{+}$ions by up to three orders of magnitude, due to electrostatic imbalance caused by the much slower diffusing $\mathrm{Na}^{+}$. Therefore, many analytical studies have adopted $\mathrm{Na}^{+}$ions as a model molecule to analyse desalination processes. We chose to use $\mathrm{Cl}^{-}$transport to provide a conservative value because $\mathrm{Cl}$ - sensitive fluorescent dyes were found to be more sensitive sensors in a recent study ${ }^{31}$.

Quantitative $\mathrm{Cl}^{-}$permeation through DOPC membrane in absence and presence of 3-LA were measured at several different inwardly directly concentration gradients (Fig. 2g and Supplementary Fig 10a). 
Based on the channel density in membrane, the single channel chloride ion permeability was calculated to be $9.5 \pm 7.5 \times 10^{-21} \mathrm{~cm}^{3} / \mathrm{s}$ for 3-LA. To provide a comparison to desalination membranes, the permeability and selectivity values for 3-LA were plotted in a water-to-salt permselectivity plot of the current polymeric desalination membranes. To provide a membrane engineering perspective, the water permeability $\left(P w, \mathrm{~cm}^{2} / \mathrm{s}\right)$ was calculated by dividing the single channel permeability $\left(\mathrm{cm}^{3} / \mathrm{s}\right)$ by the channels' projected area and further multiplied by the thickness $(\sim 4 \mathrm{~nm})$ of the membranes. As shown in Fig. 2h, the 3-LA's water-to-salt permselectivity was calculated as 1.6 $\pm 2.5 \times 10^{8}$, which overcomes the current desalination membranes' permeability-selective trade-off trendline ${ }^{10,12}$ by a factor of $\sim 10^{3}$, demonstrating good potential of using foldamer-derived AWCs for desalination membrane development.

\section{Computed water transport rates}

Atomistic molecular dynamics (MD) simulations of LA-containing 3 5 of $2.9 \mathrm{~nm}$ long, each having 26 units 1 and 26 units 2, or AQP1 embedded in solvated POPC membrane (Fig. 1c) gives water permeation rates of 3.6, 1.9, 1.2 and $0.08 \times 10^{9} \mathrm{H}_{2} \mathrm{O} / \mathrm{s}$ for $\mathbf{3}, \mathbf{4}, \mathrm{AQP} 1$ and 5 (Fig. 3a and Supplementary Movies 1-3), respectively. Although these values do not exactly match the experimental values, they do show a comparable trend, i.e., both 3-LA and 4-LA are more permeable to water than AQP1, with 5-LA being the least permeable.

\section{Proton wire breakers to block proton transport}

At first sight, the many water molecules ( $\sim 50$ inside 3 and $\sim 32$ inside 4, Fig. 3b) should readily facilitate proton transport, which nevertheless is not observed experimentally. Analysis of the Grotthuss mechanism, employing H-bonded water chain as the proton wire for proton transport, shows that every non-terminal water molecule regularly $\mathrm{H}$-bonds to others using its $\mathrm{O}$-atom and one $\mathrm{H}$-atom (Fig. 3c). In other words, non-terminal water molecules, forming no or just one $\mathrm{H}$-bond, or two $\mathrm{H}$-bonds both via only $\mathrm{O}$ - or only $\mathrm{H}$-atoms with other water molecules, can be classified as the proton wire breakers, blocking proton transport through water molecules (Fig. 3d). This is a mechanism similar to that observed in AQPs where the presence of the Asparagine Proline Alanine (NPA) motif leads to water molecule(s) in the approximate center of the channel forming up to two $\mathrm{H}$-bonds with adjacent waters using only $\mathrm{H}$-atoms, because the $\mathrm{O}$ atoms of these central waters are H-bonded by the NPA motif ${ }^{48}$. Thus, a proton is not able to hop on or off the water molecule present in this position in AQPs. Nevertheless, as suggested by few, we do acknowledge that the electrostatic effect of positively charged residues lining the pore may play an important and perhaps even predominant role in inhibiting proton passage through $\mathrm{AQPs}^{45}$. In this work, we find that the channel architecture leads to creation of a number of water molecules serving as proton wire breakers to prevent proton hopping through water molecules, and these proton wire breakers alone seem to be sufficient to achieve a high level rejection of protons. On average, about $36 \%$ of total water molecules present in $\mathbf{3}$ and $\mathbf{4}$ are found to satisfy the criteria of being proton wire breakers (Fig. 3f). One statistically meaningful snapshot in Fig. 3e illustrates 15 proton wire breakers and 20 regularly H-bonded water molecules in 4 . The Supplementary Fig. 11 highlights a snapshot of 3, having 26 proton wire breakers and 27 regularly $\mathrm{H}$-bonded water molecules.

\section{Summary}

In summary, we have presented here novel strategies for molecular

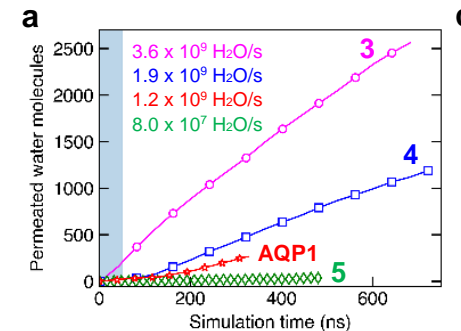

C<smiles>Oc1ccccc1</smiles>

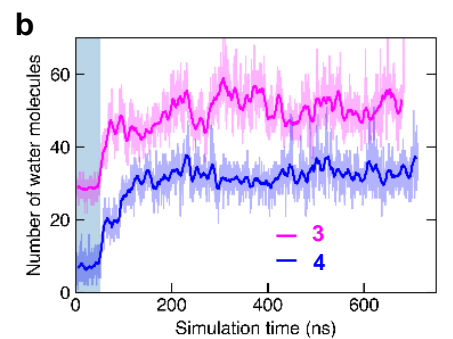

产

d<smiles>OCCOCCOO</smiles>
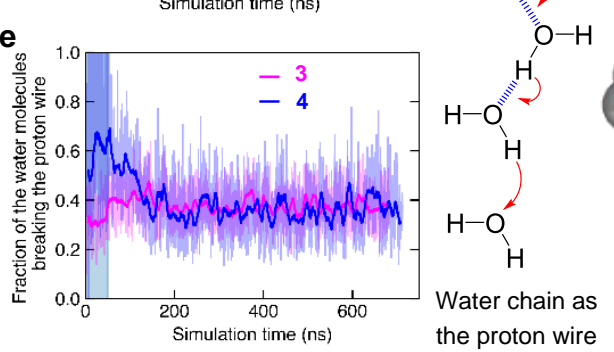

the proton wire

Fig. 3 MD-derived water transport rates and insights into proton rejection by proton wire breakers. a, Computed water transport rates of $\mathbf{3}-\mathbf{5}$ and AQP1. b, Number of water molecules inside the pores of $\mathbf{3}$ and $\mathbf{4}$. c, Proton transport through a model proton wire via the Grotthuss mechanism. d, Irregularly Hbonded water molecules, serving as proton wire breakers to block proton transport via the Grotthuss mechanism. e, Fractions of proton wire breakers over all water molecules inside $\mathbf{3}$ and $\mathbf{4}$.

evolution of foldamer-based ultrapermeable artificial water channels, derived from a fully $\mathrm{H}$-bonded, helically folded and pore-forming hydrazide backbone. Tuning of interior groups and attachment of lipidanchors were exploited to enhance water permeability and salt rejection. Exhibiting ultrahigh single-channel water permeability of 2.7 $\times 10^{10} \mathrm{H}_{2} \mathrm{O} / \mathrm{s}$, the most permeable water channel 4-LA further possesses high capacity to reject both salts and protons. This is the first instance of an artificial nanometer-sized water channel truly emulating the key water transport features of biological water channels. This ultrapermeable artificial aquaporin might have the potential to become the preferred candidate for fabricating next-generation membranes for water purification as well as other challenging nanobiotechnological applications where high water transport but proton and ion exclusion is required. Going beyond the specific designs presented here, we can envision a variety of foldamer-based hydrazide water channels, made up of different types of helicity codons ${ }^{32}$, having similar intermediate pore sizes as 4-LA, paralleling or even surpassing the water transport performances of 4-LA.

\section{Online content}

Any methods, additional references, Nature Research reporting summaries, source data, extended data, supplementary information, acknowledgements, peer review information; details of author 
contributions and competing interests; and statements of data availability are available.

\section{References}

Murata, K. et al. Structural determinants of water permeation through aquaporin-1. Nature 407, 599605 (2000).

2 Tajkhorshid, E. et al. Control of the Selectivity of the Aquaporin Water Channel Family by Global Orientational Tuning. Science 296, 525-530 (2002).

3 Takata, K., Matsuzaki, T. \& Tajika, Y. Aquaporins: water channel proteins of the cell membrane. Progress in histochemistry and cytochemistry 39, 1-83 (2004).

4 de Groot, B. \& Grubmüller, H. Water Permeation Across Biological Membranes: Mechanism and Dynamics of Aquaporin-1 and GlpF. Science 294, 2353-2357 (2002).

Agre, P. Aquaporin Water Channels (Nobel Lecture). Angew. Chem. Int. Ed. 43, 4278-4290 (2004)

6 Borgnia, M. J., Kozono, D., Calamita, G., Maloney, P. C. \& Agre, P. Functional reconstitution and characterization of AqpZ, the E. coli water channel protein. J. Mol. Biol. 291, 1169-1179 (1999).

7 Horner, A. et al. The mobility of single-file water molecules is governed by the number of H-bonds they may form with channel-lining residues. Sci. Adv. 1, e1400083 (2015)

8 Fane, A. G., Wang, R. \& Hu, M. X. Synthetic Membranes for Water Purification: Status and Future. Angew. Chem, Int. Ed. 54, 3368-3386 (2015).

9 Werber, J. R., Osuji, C. O. \& Elimelech, M. Materials for next-generation desalination and water purification membranes. Nat. Rev. Mater. 1 (2016)

10 Park, H. B., Kamcev, J., Robeson, L. M., Elimelech, M. \& Freeman, B. D. Maximizing the righ stuff: The trade-off between membrane permeability and selectivity. Science 356, eaab0530 (2017)

11 Hélix-Nielsen, C. Biomimetic Membranes as a Technology Platform: Challenges and Opportunities. Membranes 8, 44-59 (2018).

12 Werber, J. R. \& Elimelech, M. Permselectivity limits of biomimetic desalination membranes. Sci. Adv. 4, eaar8266 (2018).

13 Freger, V. Selectivity and polarization in water channel membranes: lessons learned from polymeric membranes and CNTs. Faraday Discuss. 209, 371-388 (2018).

14 Wagh, P. \& Escobar, I. Biomimetic and bioinspired membranes for water purification: A critical review and future directions. Environ. Prog. Sustain. 38 (2019).

15 Barboiu, M. \& Gilles, A. From Natural to Bioassisted and Biomimetic Artificial Water Channel Systems. Acc. Chem. Res. 46, 2814-2823 (2013).

16 Huo, Y. P. \& Zeng, H. Q. "Sticky"-Ends-Guided Creation of Functional Hollow Nanopores for Guest Encapsulation and Water Transport. Acc. Chem. Res. 49, 922-930 (2016).

17 Gong, B. Artificial water channels: inspiration, progress, and challenges. Faraday Discuss. 209, 415-427 (2018)

18 Song, W. \& Kumar, M. Artificial water channels: toward and beyond desalination. Curr. Opin. Chem. Eng. 25, 9-17 (2019).

19 Bhushan, B. Biomimetics: lessons from nature-an overview. Phil. Trans. R. Soc. A. 367, 1445-1486 (2009).

20 Doyle, D. A. et al. The structure of the potassium channel: molecular basis of $\mathrm{K}^{+}$conduction and selectivity. Science 280, 69-77 (1998).

21 Yu, F. H. \& Catterall, W. A. Overview of the voltage-gated sodium channel family. Genome Biol. 4, 207 (2003).

22 Reuter, H. A variety of calcium channels. Nature 316, 391 (1985).

23 Dutzler, R., Campbell, E. B. \& MacKinnon, R. Gating the Selectivity Filter in ClC Chloride Channels. Science 300, 108-112 (2003).

24 Mould, J. A. et al. Mechanism for Proton Conduction of the M2 Ion Channel of Influenza A Virus. J. Biol. Chem. 275, 8592-8599 (2000).

25 Kaucher, M. S. et al. Selective transport of water mediated by porous dendritic dipeptides. J. Am Chem. Soc. 129, 11698-11699 (2007).

26 Zhou, X. B. et al. Self-assembling subnanometer pores with unusual mass-transport properties. Nat. Commun. 3, 949 (2012).

27 Hu, C. B., Chen, Z. X., Tang, G. F., Hou, J. L. \& Li, Z. T. Single-Molecular Artificial Transmembrane Water Channels. J. Am. Chem. Soc 134, 8384-8387 (2012).

28 Licsandru, E. et al. Salt-Excluding Artificial Water Channels Exhibiting Enhanced Dipolar Water and Proton Translocation. J. Am. Chem. Soc. 138, 5403-5409 (2016).

29 Zhao, H. Q., Sheng, S., Hong, Y. H. \& Zeng, H. Q. Proton Gradient-Induced Water Transport Mediated by Water Wires inside Narrow Aquapores of Aquafoldamer Molecules J. Am. Chem. Soc. 136, 14270-14276 (2014)

30 Shen, Y.-x. et al. Highly permeable artificial water channels that can self-assemble into twodimensional arrays. Proc. Natl. Acad. Sci. USA 112, 9810-9815 (2015).

31 Song, W. et al. Artificial water channels enable fast and selective water permeation through waterwire networks. Nature Nanotech. 15, 73-79 (2020).

32 Shen, J. et al. Polypyridine-Based Helical Amide Foldamer Channels: Rapid Transport of Water and Protons with High Ion Rejection. Angew. Chem. Int. Ed. 59, DOI: 10.1002/anie.202003512 (2020).

33 Shen, J. et al. Aquafoldmer-Based Aquaporin-like Synthetic Water Channel. J. Am. Chem. Soc. 142, 10050-10058 (2020)

34 Tunuguntla, R. H. et al. Enhanced water permeability and tunable ion selectivity in subnanometer carbon nanotube porins. Science 357, 792-796 (2017).

35 Gale, P. A., Davis, J. T. \& Quesada, R. Anion transport and supramolecular medicinal chemistry. Chem. Soc. Rev. 46, 2497-2519 (2017).

36 Sakai, N. \& Matile, S. Synthetic Ion Channels. Langmuir 29, 9031-9040 (2013).

37 Si, W., Xin, P., Li, Z.-T. \& Hou, J.-L. Tubular Unimolecular Transmembrane Channels Construction Strategy and Transport Activities. Acc. Chem. Res. 48, 1612-1619 (2015).

38 Howorka, S. Building membrane nanopores. Nat. Nanotech. 12, 619-630 (2017).

39 Zheng, S.-P., Huang, L.-B., Sun, Z. \& Barboiu, M. Self-assembled Artificial Ion-Channels toward Natural Selection of Functions. Angew. Chem. Int. Ed. 59, DOI: 10.1002/anie.201915287 (2020).

40 Roy, A. et al. Polyhydrazide-Based Organic Nanotubes as Efficient and Selective Artificial Iodide Channels. Angew. Chem. Int. Ed. 59, 4806-4813 (2020).

41 Tunuguntla, R. H., Allen, F. I., Kim, K., Belliveau, A. \& Noy, A. Ultrafast proton transport in sub1-nm diameter carbon nanotube porins. Nat. Nanotech. 11, 639-644 (2016).
42 Ren, C. et al. Molecular Swings as Highly Active Ion Transporters. Angew. Chem. Int. Ed. 58, 8034 8038 (2019).

43 Zeng, L. Z., Zhang, H., Wang, T. \& Li, T. Enhancing $\mathrm{K}^{+}$transport activity and selectivity of synthetic $\mathrm{K}^{+}$channels via electron-donating effects. Chem. Commun. 56, 1211-1214 (2020).

44 Chen, F. et al. Pyridine/Oxadiazole-Based Helical Foldamer Ion Channels with Exceptionally High $\mathrm{K}^{+} / \mathrm{Na}^{+}$Selectivity. Angew. Chem. Int. Ed. 59, 1440-1444 (2020).

45 de Groot, B., Frigato, T., Helms, V. \& Grubmüller, H. The Mechanism of Proton Exclusion in the Aquaporin-1 Water Channel. J. Mol. Biol. 333, 279-293 (2003).

46 Pohl, P., Saparov, S. M., Borgnia, M. J. \& Agre, P. Highly selective water channel activity measure by voltage clamp: Analysis of planar lipid bilayers reconstituted with purified AqpZ. Proc. Natl. Acad. Sci. USA 98, 9624-9629 (2001).

47 Pham, T. A. et al. Salt Solutions in Carbon Nanotubes: The Role of Cation- $\pi$ Interactions. J. Phys Chem. C 120, 7332-7338 (2016).

48 Kosinska Eriksson, U. et al. Subangstrom Resolution X-Ray Structure Details Aquaporin-Water Interactions. Science 340, 1346-1349 (2013)

49 Horner, A., Siligan, C., Cornean, A. \& Pohl, P. Positively charged residues at the channel mouth boost single-file water flow. Faraday Discuss. 209, 55-65 (2018).

50 Ren, C. et al. A halogen bond-mediated highly active artificial chloride channel with high anticance activity. Chem. Sci. 9, 4044-4051 (2018).

\section{Methods}

\section{Typical polymerization conditions}

In a $20 \mathrm{~mL}$ reaction vial, 2-alkoxyisophthalic acid (1a - 1b, $0.118 \mathrm{mmol})$ and 4,6-bis(octyloxy)isophthalohydrazide $(2,0.118 \mathrm{mmol})$ were introduced. Coupling reagents $(0.354 \mathrm{mmol})$ were added to each reaction vial. This was followed by adding freshly distilled $\mathrm{CH}_{2} \mathrm{Cl}_{2}(5 \mathrm{~mL})$ and $1 \mathrm{~mL}$ DMF (dimethylformamide) in a $\mathrm{N}_{2}$ atmosphere. $100 \mu \mathrm{L}$ DIEA (N,N-Diisopropyl ethylamine) was then added in the reaction mixture, and the solution was stirred for 2 days at room temperature. After completion of reaction, solvent was evaporated to remove $\mathrm{CH}_{2} \mathrm{Cl}_{2}$ and DMF. The obtained residue was first washed with $10 \mathrm{~mL} \mathrm{MeOH} / \mathrm{H}_{2} \mathrm{O}(1: 1)$ and subsequently washed with $10 \mathrm{~mL}$ water and $10 \mathrm{~mL} \mathrm{MeOH}$ and dried in oven $\left(60^{\circ} \mathrm{C}\right)$ to obtain polymers 3-5 as off-white solid powder with yields of $60-80 \%$. Average molecular weights $\left(M_{n}\right)$ of all these polymers were determined by Gel Permeation Chromatography.

\section{MALDI-TOF measurement}

MALDI-TOF mass spectra were acquired with Bruker Ultraflextreme (Bruker Daltonik GmbH, Germany) equipped with Bruker smartbeam II $355 \mathrm{~nm}$ nitrogen laser with an accelerating voltage of $25 \mathrm{kV}$ in the linear configuration. Mass spectra were measured by using the positive mode of mass spectroscopy using reported protocol. The matrix used in the experiment was trans-2-[3-(4tert-Butylphenyl)-2-methyl-2-propenylidene]malononitrile (DCTB) from TCI and used directly without further purification. Selection of the laser used for ionization was performed directly through the software and required no adjustments to the individual lasers. MALDI-TOF experiments were carried out for polymers 3 and $\mathbf{4}$ and the instrument could detect up to 10,000 Da. Both compounds displayed characteristic mass pattern of repeating unit in the MALDI spectra. The interior of the helical channel is highly electron rich and therefore can bind with multiple metal ions such as $\mathrm{Na}^{+}$or $\mathrm{K}^{+}$ions to give rise to doubly or multiply charged species and all these peaks can be observed in the MALDI spectra.

\section{Water transport study using a stopped-flow instrument}

1,2-dioleoyl-sn-glycero-3-phosphocholine (DOPC, $0.24 \mathrm{ml}, 25 \mathrm{mg} / \mathrm{mL}$ in $\mathrm{CHCl}_{3}$, Avanti Polar Lipids, USA) and channel samples (3 - 5 or gA in chloroform) were mixed at different lipid to channel molar ratios (1500:1 to 9000:1) in micro-tubes $(2 \mathrm{ml})$. The solvent was removed by $\mathrm{N}_{2}$ flow and the resulting film was dried under high vacuum overnight. HEPES buffer $(10 \mathrm{mM}$ HEPES, $100 \mathrm{mM} \mathrm{NaCl}, \mathrm{pH}=7.0,1.0 \mathrm{~mL}$ ) was then added, followed by vortexing the solution for $30 \mathrm{~s}$ and then ten cycles of sonication $(37 \mathrm{kHz}$, power $100,70{ }^{\circ} \mathrm{C}, 2.5 \mathrm{~min}$ ) in order to maximize incorporation extent of channel molecules into membrane. A glass spatula was used if necessary to make sure the residue was fully detached from the surface of the micro-tube. The mixture was further subjected to 10 freeze-thaw cycles (freezing in liquid $\mathrm{N}_{2}$ for $1 \mathrm{~min}$ and heating $55^{\circ} \mathrm{C}$ water bath for $2 \mathrm{~min}$ ), and extruded at $80^{\circ} \mathrm{C}$ for 15 times. The LUVs obtained this way contained $6 \mathrm{mg} / \mathrm{mL}$ of lipids and were stored in a $4{ }^{\circ} \mathrm{C}$ fridge before use, and diluted six times with HEPES buffer to make $1 \mathrm{mg} / \mathrm{mL}$ LUV for stopped flow measurements. The particle size of LUV $(120 \mathrm{~nm})$ was 
characterized by dynamic light scattering (Zetasizer Nano, Malvern Instruments Ltd., UK). The water permeability measurements were conducted on a stoppedflow instrument (Chirascan Circular Dichroism Spectrometer, Applied Photophysics, UK). Exposure of vesicles to three types of hypertonic osmolytes resulted in the shrinkage of the vesicles due to an outwardly directed osmotic gradient. The abrupt decrease of the vesicle size leads to an increase in light scattering intensity at $90^{\circ}$ angle based on the Rayleigh-Gans theory. The changes of light scattering intensity caused by vesicle shrinkage were recorded at a wavelength of $577 \mathrm{~nm}$, and were fitted in the following form of single exponential function.

$$
y=A^{*} \exp (-k x)+y 0
$$

where $\mathrm{y}=$ change in the light scattering, $k$ is the exponential coefficient of the change in the light scattering and $\mathrm{x}$ is time.

With the assumption that change in the light scattering intensity is proportional to the change in the vesicle volume $\left(\Delta \mathrm{V} / \mathrm{V}_{0}\right)$ based on the Boylevan't Hoff law, the osmotic permeability $\left(P_{f}\right)$ in the unit of $\mathrm{cm} / \mathrm{s}$ was calculated as follow:

$$
P_{f}=k /\left(\left(S / V_{0}\right) * V_{W} \mathrm{x} \Delta_{o s m}\right)
$$

where $k$ is the exponential coefficient of the change in the light scattering; $S$ and $V_{0}$ are the initial surface area and volume of the vesicles, respectively; $V_{w}$ is the molar volume of water, and $\Delta_{o s m}$ is the osmolarity difference.

To calculate the true water permeability $\left(P_{s c}\right.$ in the unit of $\left.\mathrm{cm}^{3} / \mathrm{s}\right)$ of water channels, the $P_{f(\text { blank })}$ value of the blank vesicle without water channels needs to be deducted from $P_{f(\text { (channel) }}$, which was multiplied by the vesical surface area $(S)$ and divided by the number of water channels $(N)$ incorporated in the liposome as shown below.

$$
P_{s c}=\left(P_{f(\text { channel })}-P_{f(b l a n k)}\right) *(S / \mathrm{N})
$$

\section{Determination of channel insertion efficiency}

In order to calculate channel insertion efficiency of the water channels in the lipid bilayer membrane, a calibration curve was first created. At first, blank LUVs (without any synthetic channels) were prepared from $3 \mathrm{mg} / \mathrm{mL}$ to 7 $\mathrm{mg} / \mathrm{mL}$ concentration by using the film rehydration method (without extrusion) as discussed above. All these solutions were scanned on a UV-Vis spectrometer and UV absorbance was found to be decreased proportionally with decrease in concentration. Calibration curve was plotted using this UV-Vis data at $280 \mathrm{~nm}$. Blank LUVs were also prepared by extruding through $200 \mathrm{~nm}$ membrane for 15 times to obtain monodispersed unilamellar vesicles with an initial concentration of $6 \mathrm{mg} / \mathrm{mL}$ which is equivalent to our experimental condition. UV absorbance was recorded with these extruded blank liposomes at $280 \mathrm{~nm}$ wavelength. The obtained data was used to get actual lipid concentration i.e. $4.4 \pm 0.7 \mathrm{mg} / \mathrm{mL}$ and around $20 \%$ lipid was lost during extrusion process.

Since the concentration of synthetic water channels is extremely low as compared to lipid concentration in our experimental condition, the UVabsorbance plots of lipid were found to be superimposed with the absorbance values of channels. Therefore, we measured fluorescence spectra of channelcontaining liposomes ( $\lambda_{\mathrm{ex}}=310 \mathrm{~nm}, \lambda_{\mathrm{em}}=430 \mathrm{~nm}$ for channel molecules). In these experiments, two separate batches of liposomes, embedded with artificial channels were prepared using film rehydration method as discussed earlier for each concentration ratio. One of the batches were not extruded and other batch was extruded through $200 \mathrm{~nm}$ membrane for 15 times to obtain monodispersed LUVs. Fluorescence spectra were recorded for all these channel-containing liposomes. Considering the lipid loss from the UV-Vis experiment and the ratio between fluorescence intensities (at $\lambda_{\mathrm{em}}=430 \mathrm{~nm}$ ) of non-extruded and extruded liposomes provided us the value of insertion efficiency of the artificial water channels in the lipid membrane. The obtained insertion efficiency values were further used to calculate actual single channel water permeability of artificial water channels.

\section{Activation energy measurements}

To determine activation energies for water transport, water transport kinetics was measured by using stopped-flow instrument from $6{ }^{\circ} \mathrm{C}$ to $25^{\circ} \mathrm{C}$. For these experiments, temperature of the solution reservoir and the measurement cell of the stopped-flow instrument were maintained by a recirculating heater/chiller (Polystat, Cole Parmer). Light scattering kinetic rates representing water transport through artificial water channels at varying temperatures were fitted into the following Arrhenius equation to obtain activation energy $\left(E_{a}\right)$.

$$
\ln (k)=\ln (A)-E_{\alpha} /\left(R^{*} T\right)
$$

where $k$ is the exponential coefficient of the change in the light scattering; $\mathrm{A}$ is pre-exponential factor; $E_{\alpha}$ is activation energy; $\mathrm{R}$ is gas constant; $\mathrm{T}$ is absolute temperature.

\section{Chloride ion permeability measurement}

DOPC and 3-LA reconstituted DOPC liposomes were prepared using the same method of preparing the samples for water permeability measurements except for that different rehydration buffer composition was used (1 mM lucigenin, 10 $\mathrm{mM}$ HEPES, $200 \mathrm{mM} \mathrm{KNO}{ }_{3}, 10 \mu \mathrm{M}$ valinomycin at $\mathrm{pH}$ 7.0). $\mathrm{K}^{+}$ionophore valinomycin was added to mitigate the electrostatic imbalance caused by $\mathrm{Cl}$ permeation. After extrusion, the reconstituted vesicles were purified by Sephadex G-50 columns to remove free (unencapsulated) lucigenin dyes. The vesicles were exposed to the $\mathrm{Cl}^{-}$-containing buffer in the stopped-flow equipment, and quantitative $\mathrm{Cl}^{-}$flux was calculated by the Stern-Volmer equation as shown in the following.

$$
\frac{F}{F_{0}}-1=K_{s v}\left[\mathrm{Cl}^{-}\right]
$$

where $\mathrm{F}$ is fluorescence intensity change inside the vesicles over time, $\mathrm{F}_{0}$ is the initial fluorescence intensity at $\mathrm{Cl}^{-}$free condition which correspond to the initial $(t=0)$ fluorescence intensity from the stopped-flow experiments, $\left[\mathrm{Cl}^{-}\right]$is the molar concentration of the chloride inside the vesicles, and $\mathrm{K}_{\mathrm{sv}}$ is the dye SternVolmer constant. The $\mathrm{K}_{\mathrm{sv}}$ of the dye sensor within DOPC vesicles was experimentally determined as shown in Supplementary Fig. 10b. Permeability calculation was performed by utilizing the first derivative of the Stern-Volmer equation with respect to time (at times approaching zero) as shown below.

$$
\left.\frac{d\left[C l^{-}\right]}{d t}\right|_{t=0}=\left.\frac{-1}{K_{s v} F_{0}} \frac{d F}{d t}\right|_{t=0}
$$

From the left side of the equation, quantitative $\mathrm{Cl}^{-}$concentration change was calculated and influx of $\mathrm{Cl}^{-}$through the vesicular membranes were calculated based on the size of the vesicles. The measurements were repeated at different concentration gradient conditions and the permeability coefficients were calculated from these data set.

\section{Fluorescence correlation spectroscopy (FCS) to determine channel density of 3-LA in lipid membranes for the calculation of single channel $\mathrm{Cl}^{-}$permeability}

3-LA channels were labeled with 5-(and-6-)-((N-(5-aminopentyl)amino) carbonyl)tetramethylrhodamine fluorescence dyes using dicyclohexylcarbodiimide crosslinkers, by mixing the channels, dyes, and crosslinkers at a molar ratio of 1:10:10 in dimethyl sulfoxide (DMSO) for 24 hours at room temperature using a previously used procedure (REF). Unreacted free dyes were removed by dialysis with fresh DMSO. These dye-labeled channels were then used to prepare the 3-LA reconstituted DOPC vesicles, and these vesicles were subjected to FCS experiments. The time dependent fluorescence fluctuation inside the confocal volume was monitored using a Zeiss Laser Scanning Confocal microscope (LSM 880) 880 equipped with the Airyscan detector. A DPSS laser with an excitation wavelength at $561 \mathrm{~nm}, 20 \mathrm{~mW}$ was conducted to measure the emission intensity in the wavelength range from $570 \mathrm{~nm}$ to $695 \mathrm{~nm}$. The average number of vesicles and micelles was measured for 10 seconds, obtaining 10 counts and through 3 repeated FCS measurements. The collected autocorrelation curves, $G(\tau)$, were fitted with the triplet state and translation parameters using the ZEN software (black edition). 


$$
G(\tau)=\frac{1}{N} \sum_{i=0}^{M} f_{i}\left[\frac{1}{1+\tau / \tau_{D_{i}}}\right]\left[\frac{1}{1+(r / z)^{2}\left(\tau / \tau_{D_{i}}\right)}\right]^{1 / 2}
$$

where $\tau$ is the lag time, $r$ and $z$ are the radius and the half height of the confocal space, $\tau_{D_{i}}$ is the lateral diffusion time of fluorescence species $i$, and the $N$ is the average number of fluorescence species in the confocal space. From this function, the number of 3-LA reconstituted vesicles were extracted $\left(N_{\text {ves }}\right)$. After that, the same vesicle samples were completely micellized by adding a final concentration of $4 \%(\mathrm{w} / \mathrm{v})$ octyl $\beta$-d-glucoside (OG) and the sample analysis was repeated to obtain the average number of micellized channels $\left(N_{\text {channel }}\right)$. From the ratio of $N_{\text {channel }}$ to $N_{\text {ves }}$, the channel number density in the DOPC vesicular membranes were determined (Supplementary Fig. 10c).

\section{The HPTS assay for cation transport study under high salt gradient}

Egg yolk L- $\alpha$-phosphatidylcholine (EYPC, $1 \mathrm{ml}, 25 \mathrm{mg} / \mathrm{mL}$ in $\mathrm{CHCl}_{3}$, Avanti Polar Lipids, USA) lipid samples were added to a $10 \mathrm{~mL}$ round bottomed flask and $\mathrm{CHCl}_{3}$ was evaporated by slowly purging $\mathrm{N}_{2}$. After drying the resulting thin film under high vacuum overnight at room temperature, the film was hydrated with a HEPES buffer solution $(1 \mathrm{~mL}, 10 \mathrm{mM}$ HEPES, $\mathrm{pH}=7.0)$ containing the pH sensitive HPTS dye (8-hydroxypyrene-1,3,6-trisulfonic acid, $0.5 \mathrm{mM}$ ) at room temperature for 1 hour (with occasional vortexing after every 15 minutes) to obtain a milky suspension. The mixture was then subjected to 10 freeze-thaw cycles (freezing in liquid $\mathrm{N}_{2}$ for 1 minute and heating at $55^{\circ} \mathrm{C}$ in water bath for 2 minutes). The vesicle suspension was extruded through polycarbonate membrane $(0.1 \mu \mathrm{m})$ to produce monodispersed LUVs of about $100 \mathrm{~nm}$ in diameter with HPTS encapsulated inside. The extravesicular HPTS dye was removed by using size exclusion chromatography (stationary phase: Sephadex G-50, GE Healthcare, USA, mobile phase: $10 \mathrm{mM}$ HEPES buffer, $\mathrm{pH}=7.0$ ) and diluted with the mobile phase to yield $3.2 \mathrm{~mL}$ of $10 \mathrm{mM}$ lipid stock solution.

$30 \mu \mathrm{L}$ of HPTS-containing LUVs was added to $1970 \mu \mathrm{L}$ of HEPES buffer (10 mM HEPES, $200 \mathrm{mM} \mathrm{M}_{2} \mathrm{SO}_{4}, \mathrm{pH}=8.0$ where $\mathrm{M}^{+}=\mathrm{Na}^{+}$and $\mathrm{K}^{+}$) in a clean fluorescence cuvette to generate a salt gradient across lipid bilayer. This cuvette was placed on the fluorescence instrument (at $t=0 \mathrm{~s}$ ) equipped with magnetic stirrer. Fluorescence emission intensity of HPTS dye, $F_{\mathrm{t}}$ was monitored at $\lambda_{\mathrm{em}}=$ $510 \mathrm{~nm}\left(\lambda_{\mathrm{ex}}=450 \mathrm{~nm}\right)$ with time. Channels of various types $(1 \mu \mathrm{M}$, dissolved in DMF) was added at $t=70 \mathrm{~s}$ and recorded simultaneously for 300 seconds using fluorescence spectrophotometer (Hitachi, Model F-7100, Japan). Finally at $t=370 \mathrm{~s}, 20 \mu \mathrm{L}$ of $20 \%$ Triton X-100 was added to destroy all vesicles which resulted in destruction of $\mathrm{pH}$ gradient to achieve the maximum change in fluorescence emission intensity of HPTS dye. Fluorescence data was also recorded for gramicidin $\mathrm{A}(\mathrm{gA}, 2 \mu \mathrm{M})$ under same experimental condition and the obtained activity was compared with artificial water channels.

The time axis was offset according to the following equation: $t=t-70$. Fluorescence intensities $\left(F_{\mathrm{t}}\right)$ were normalized to fractional emission intensity $I_{\mathrm{F}}$ using following equation:

$$
I_{\mathrm{F}}=\left[\left(F_{\mathrm{t}}-F_{0}\right) /\left(F_{\infty}-F_{0}\right)\right] \times 100
$$

whereas $F_{0}=$ Fluorescence intensity just before the compound addition (at $t=0$ s), $F_{\infty}=$ Fluorescence intensity at saturation after complete leakage (at $t=300$ $\mathrm{s})$, and $F_{\mathrm{t}}=$ Fluorescence intensity at time $t$.

\section{The HPTS assay for anion and proton transport study}

EYPC $\left(1 \mathrm{ml}, 25 \mathrm{mg} / \mathrm{mL}\right.$ in $\left.\mathrm{CHCl}_{3}\right)$ was taken in a $10 \mathrm{~mL}$ round bottomed flask and $\mathrm{CHCl}_{3}$ was evaporated by purging $\mathrm{N}_{2}$ slowly. After drying the resulting thin film under high vacuum overnight at room temperature, the film was hydrated with HEPES buffer solution ( $1 \mathrm{~mL}, 10 \mathrm{mM}$ HEPES, $100 \mathrm{mM} \mathrm{NaCl}, \mathrm{pH}=7.0$ ) containing a $\mathrm{pH}$ sensitive HPTS dye $(0.5 \mathrm{mM})$ at room temperature for 1 hour (with occasional vortexing after every 15 minutes) to obtain a milky suspension. The mixture was then subjected to 10 freeze-thaw cycles (freezing in liquid $\mathrm{N}_{2}$ for 1 minute and heating at $55{ }^{\circ} \mathrm{C}$ in water bath for 2 minutes). The vesicle suspension was extruded through polycarbonate membrane $(0.1 \mu \mathrm{m})$ to produce monodispersed LUVs of about $100 \mathrm{~nm}$ in diameter with HPTS encapsulated inside. The extravesicular HPTS dye was removed by using size exclusion chromatography (stationary phase: Sephadex G-50, GE Healthcare, USA, mobile phase: $10 \mathrm{mM}$ HEPES buffer, $\mathrm{pH}=7.0$ ) and diluted with the mobile phase to yield $3.2 \mathrm{~mL}$ of $10 \mathrm{mM}$ lipid stock solution.

$30 \mu \mathrm{L}$ of HPTS-containing LUVs was added to $1970 \mu \mathrm{L}$ of HEPES buffer (10 mM HEPES, $67 \mathrm{mM} \mathrm{K}_{2} \mathrm{SO}_{4}, \mathrm{pH}=8.0$ ) in a clean fluorescence cuvette to generate salt gradient across lipid bilayer. This cuvette was placed on the fluorescence instrument (at $t=0 \mathrm{~s}$ ) equipped with magnetic stirrer. Fluorescence emission intensity of HPTS dye, $F_{\mathrm{t}}$ was monitored at $\lambda_{\mathrm{em}}=510 \mathrm{~nm}\left(\lambda_{\mathrm{ex}}=450\right.$ $\mathrm{nm})$ with time. Channels $(\mathbf{3}, \mathbf{4}, \mathbf{5}$ and $\mathbf{L 8})$ or proton carrier (FCCP) at desired concentration in DMF was added at $t=70 \mathrm{~s}$ and recorded simultaneously for 300 seconds using fluorescence spectrophotometer (Hitachi, Model F-7100, Japan). Finally at $t=370 \mathrm{~s}, 20 \mu \mathrm{L}$ of $20 \%$ Triton X-100 was added to destroy all vesicles which resulted in destruction of $\mathrm{pH}$ gradient to achieve the maximum change in fluorescence emission intensity of HPTS dye.

The time axis was offset according to the following equation: $t=t-70$. Fluorescence intensities $\left(F_{\mathrm{t}}\right)$ were normalized to fractional emission intensity $I_{\mathrm{F}}$ using following equation:

$$
I_{\mathrm{F}}=\left[\left(F_{\mathrm{t}}-F_{0}\right) /\left(F_{\infty}-F_{0}\right)\right] \times 100
$$

whereas $F_{0}=$ Fluorescence intensity just before the compound addition (at $t=0$ $\mathrm{s}$ ), $F_{\infty}=$ Fluorescence intensity at saturation after complete leakage (at $t=300$ s), and $F_{\mathrm{t}}=$ Fluorescence intensity at time $t$.

\section{The SPQ assay for anion transport activity study}

The SPQ-containing LUV suspension $\left(30 \mu \mathrm{L}, 10 \mathrm{mM}\right.$ in $\left.225 \mathrm{mM} \mathrm{NaNO}_{3}\right)$ was added to a $\mathrm{NaCl}$ solution $(1.7 \mathrm{~mL}, 225 \mathrm{mM})$ to create an extravesicular chloride gradient. A solution of channel molecule 3-LA, 4-LA and 5-LA $(1 \mu \mathrm{M})$ or anion channel L8 $(6 \mu \mathrm{M})$ in DMF at different concentrations was then injected into the suspension under gentle stirring. Upon the addition of channels, the emission of SPQ was immediately monitored at $430 \mathrm{~nm}$ with excitations at 360 $\mathrm{nm}$ for 300 seconds using fluorescence spectrophotometer (Hitachi, Model F7100 , Japan $)$ after which time an aqueous solution of Triton X-100 (20 $\mu \mathrm{L}, 20 \%$ $\mathrm{v} / \mathrm{v}$ ) was immediately added to completely destruct the chloride gradient. The final transport trace was obtained by normalizing the fluorescence intensity using the following equation:

$$
I_{\mathrm{F}}=\left[\left(F_{\mathrm{t}}-F_{0}\right) /\left(F_{\infty}-F_{0}\right)\right]
$$

Where $F_{0}=$ Fluorescence intensity just before the compound addition (at $t=0$ s), $F_{\mathrm{t}}=$ Fluorescence intensity at time $t$, and $F_{\infty}=$ Fluorescence intensity at saturation after complete leakage (at $t=300 \mathrm{~s}$ ).

\section{Molecular dynamics simulations}

All MD simulations were performed using the MD program NAMD2 ${ }^{51}$, periodic boundary conditions and particle mesh Ewald (PME) method to calculate the long range electrostatics ${ }^{52}$. The Nose-Hoover Langevin piston ${ }^{53,54}$ and Langevin thermostat ${ }^{55}$ were used to maintain the constant pressure and temperature in the system. CHARMM36 force field parameters ${ }^{56}$ described the bonded and nonbonded interactions of among, lipid bilayer membranes, water and ions. A 810-12 ̊ cutoff scheme was used to calculate van der Waals and short range electrostatics forces. All simulations were performed using a 2 fs time step to integrate the equation of motion. SETTLE algorithm ${ }^{57}$ was applied to keep water molecules rigid whereas RATTLE algorithm ${ }^{58}$ constrained all other covalent bonds involving hydrogen atoms. The coordinates of the system were saved at an interval of $19.2 \mathrm{ps}$. The analysis and post processing the simulation trajectories were performed using $\mathrm{VMD}^{59}$ and CPPTRAJ ${ }^{60}$.

The initial PDB structures of LA-modified channels 3-5 were created using Gaussview ${ }^{61}$. The topology and force field parameters for the monomeric unit of the channel (along with all three variant 3,4,5) and LA anchor were obtained using the CHARMM general force fields (CGenFF) webserver. ${ }^{62}$ We connected 
the LA anchors to the terminal residues and obtained the protein structure file (psf) of the channels using the psfgen tool of VMD. The channels were then embedded into a $9 \times 9 \mathrm{~nm}^{2}$ patch of pre-equilibrated POPC lipid bilayer membrane. The lipid patch was generated using the CHARMM-GUI membrane builder $^{63}$ and pre-equilibrated for approximately $400 \mathrm{~ns}$. Lipid molecules that overlapped with the channel were removed. The system was then solvated with water ${ }^{64}$ using the Solvate plugin of VMD. Sodium and chloride ions were added to $1 \mathrm{M}$ concentration using the Autoionize plugin of VMD. The final assembled system measured $9 \times 9 \times 9 \mathrm{~nm}^{3}$ and contained approximately 80,000 atoms.

Following the assembly, the system underwent 1200 steps of energy minimization using the conjugate gradient method to remove steric clashes. After energy minimization, the system was subjected to a $50 \mathrm{~ns}$ equilibration at a constant number of atoms $(\mathrm{N})$, pressure $(\mathrm{P}=1 \mathrm{bar})$ and temperature $(\mathrm{T}=300$ $\mathrm{K})$, the NPT ensemble, with harmonic restraints applied to all non-hydrogen atoms of channels that surrounded the transmembrane pore. The restraints were applied relative to the initial coordinates of the atoms, the spring constants were $1 \mathrm{kcal} \mathrm{mol}^{-1} \AA^{-2}$. After $50 \mathrm{~ns}$, the harmonic restraints were removed, and the system was equilibrated free of any restraints for $\sim 700 \mathrm{~ns}$.

\section{Reporting summary}

Further information on research design is available in the Nature Research Reporting Summary linked to this paper.

\section{Data availability}

The datasets that support the finding of this study are available in figshare repository with the identifier(s) (https://figshare.com/s/0354959049b2c0ed4c61)

\section{References}

51 Phillips, J. C. et al. Scalable molecular dynamics with NAMD. J. Comput. Chem. 26, 1781-1802 (2005).

52 Darden, T., York, D. \& Pedersen, L. Particle mesh ewald - An N.log(N) method for ewald sums in large systems. J. Chem. Phys. 98, 10089-10092 (1993).

53 Feller, S. E., Zhang, Y., Pastor, R. W. \& Brooks, B. R. Constant pressure molecular dynamics simulation: the Langevin piston method. J. Chem. Phys. 103, 4613-4621 (1995).

54 Martyna, G. J., Tobias, D. J. \& Klein, M. L. Constant pressure molecular dynamics algorithms. The J. Chem. Phys. 101, 4177-4189 (1994).

55 Sindhikara, D. J., Kim, S., Voter, A. F. \& Roitberg, A. E. Bad seeds sprout perilous dynamics: Stochastic thermostat induced trajectory synchronization in biomolecules. J. Chem. Theory Comput 5, 1624-1631 (2009).

56 Klauda, J. B. et al. Update of the CHARMM all-atom additive force field for lipids: validation on six lipid types. J. Chem. Phys. B 114, 7830-7843 (2010).

57 Miyamoto, S. \& Kollman, P. A. Settle: An analytical version of the SHAKE and RATTLE algorithm for rigid water models. J. Comput. Chem. 13, 952-962 (1992).

58 Andersen, H. C. Rattle: A "velocity" version of the shake algorithm for molecular dynamics calculations. J. Comput. Phys. 52, 24-34 (1983).

59 Humphrey, W., Dalke, A. \& Schulten, K. VMD: Visual molecular dynamics. J. Mol. Graph. 14, 3338 (1996).

60 Roe, D. R. \& Cheatham, T. E., III. PTRAJ and CPPTRAJ: Software for Processing and Analysis of Molecular Dynamics Trajectory Data. J. Chem. Theory Comput. 9, 3084-3095 (2013).

61 Gaussian 16 Rev. C.01 (Wallingford, CT, 2016)

62 Vanommeslaeghe, K. et al. CHARMM general force field: A force field for drug-like molecules compatible with the CHARMM all-atom additive biological force fields. J. Comput. Chem. 31, 671 690 (2010).

63 Jo, S., Kim, T., Iyer, V. G. \& Im, W. CHARMM-GUI: a web-based graphical user interface for CHARMM. J. Comput. Chem. 29, 1859-1865 (2008).

64 Jorgensen, W. L., Chandrasekhar, J., Madura, J. D., Impey, R. W. \& Klein, M. L. Comparison of simple potential function for simulating liquid water. J. Chem. Phys. 79, 926-935 (1983).

Acknowledgements This study was supported by NanoBio Lab (Biomedical Research Council, Agency for Science, Technology and Research, Singapore), the Singapore National Research Foundation under its Environment and Water Research Programme and administered by PUB, the National Science Foundation (USA) under grant DMR-1827346, the National Institutes of Health under grant P41-GM104601. Supercomputer time was provided through the Early Allocation grant on Frontera (FTA-Chemla), XSEDE Allocation Grant no. MCA05S028 and the Blue Waters petascale supercomputer system at the University of Illinois at Urbana-Champaign. This work was also supported by the National Science Foundation grants CBET-1946392 and CBET- 1952295 to MK

Author contributions A.R. synthesized polymers $\mathbf{3}$ and $\mathbf{5}$ and conducted water/ion transport study; J.S. synthesized polymers $\mathbf{4}$ and conducted water/ion transport study; H.J. and A.A. performed MD study; W.S., Y.T. and M.K. determined water over chloride selectivity; R.Y., N.L. and C.R. performed some ion transport study; H.Z. conceived the project and wrote the manuscript with input from A.R. and M.K.. All authors edited the manuscript.

Competing interests The authors declare no competing interests.
Additional information

Supplementary information is available for this paper at https://doi.org/xxxxxx. Correspondence and requests for materials should be addressed to H.Z.

Reprints and permissions information is available at http://www.nature.com/reprints. 